\title{
Numerical approximation of vector-valued highly oscillatory integrals
}

\author{
Sheehan Olver *
}

\begin{abstract}
We present a method for the efficient approximation of integrals with highly oscillatory vector-valued kernels, such as the Airy function. We generalize the asymptotic expansion for the vector-valued case, which allows us to determine the asymptotic order of a Levin-type method. Levin-type methods are constructed using collocation, and choosing the basis wisely results in an approximation with significantly higher asymptotic order.
\end{abstract}

\section{Introduction}

We are concerned with the integral

$$
I[\boldsymbol{f}]=\int_{a}^{b} \boldsymbol{f}(x)^{\top} \boldsymbol{y}(x) \mathrm{d} x,
$$

where $\boldsymbol{f}$ is a smooth vector-valued function and $\boldsymbol{y}$ is a highly oscillatory vector-valued function. We assume that $\boldsymbol{y}$ depends on a parameter $\omega$ that determines the frequency of oscillations. We also assume that $\boldsymbol{y}$ satisfies the differential equation

$$
\boldsymbol{y}^{\prime}(x)=A(x) \boldsymbol{y}(x)
$$

where $A$ is a matrix-valued function that depends on $\omega$. Some common examples include:

$$
\begin{array}{ll}
y(x)=\mathrm{e}^{\mathrm{i} \omega g(x)}, & A(x)=\mathrm{i} \omega g^{\prime}(x), \\
\boldsymbol{y}(x)=\left(\begin{array}{c}
J_{m-1}(\omega x) \\
J_{m}(\omega x)
\end{array}\right), & A(x)=\left(\begin{array}{cc}
\frac{m-1}{x} & -\omega \\
\omega & -\frac{m}{x}
\end{array}\right), \\
\boldsymbol{y}(x)=\left(\begin{array}{c}
\mathrm{Ai}(-\omega x) \\
-\omega \mathrm{Ai}^{\prime}(-\omega x)
\end{array}\right), & A(x)=\left(\begin{array}{cc}
0 & 1 \\
-\omega^{3} x & 0
\end{array}\right),
\end{array}
$$

where Ai is an Airy function and $J_{m}$ is a Bessel function [6].

For large values of $\omega$, traditional quadrature techniques fail to approximate $I[\boldsymbol{f}]$ efficiently. Unless the number of sample points is sufficiently greater than the number of oscillations, Gauss-Legendre quadrature gives an approximation which is essentially random. In the one-dimensional case of $y=\mathrm{e}^{\mathrm{i} \omega g}$ with no stationary points, the integral $I[f]$ is $O\left(\omega^{-1}\right)$ for increasing $\omega[\mathbf{9}]$. This compares with an error of order $O(1)$ of the traditional techniques. This implies that it is more accurate to approximate $I[f]$ by zero than to use Gauss-Legendre quadrature!

The goal of this paper is to generalize a method developed by Levin in [5] to obtain higher asymptotic orders. This will be accomplished in a similar vein to $[\mathbf{7}]$, which dealt with the case of $y=\mathrm{e}^{\mathrm{i} \omega g}$. In $[\mathbf{7}]$, the asymptotic expansion was used to determine the asymptotic behaviour of the error of a Levin-type method. Thus our first task is to derive a vector-valued kernel version of the asymptotic expansion. This is accomplished in Section 3, using the asymptotic tools developed in Section 2. With an asymptotic expansion in hand, we can successfully prove the order of error for a Levin-type method in Section 4. In [7], it was noted that choosing a certain basis causes the asymptotic order to increase without the need for nontrivial multiplicities. In Section 5, we construct a vector-valued version of such a basis, allowing us to obtain higher asymptotic orders with significantly smaller systems.

* Department of Applied Mathematics and Theoretical Physics, Centre for Mathematical Sciences, Wilberforce Rd, Cambridge CB3 0WA, UK, email: S.0lver@damtp.cam.ac.uk 


\section{Matrix and function asymptotics}

In this section we present notation for the asymptotic behaviour of matrices and functions that depend on $\omega$ as a parameter. For the entirety of the paper, all norms are $L^{\infty}$ norms, for vectors, matrices and functions. The norm of a function is taken over the interval $[a, b]$. Furthermore, we use $\mathbf{1}_{n \times m}$ to represent an $n \times m$ matrix whose entries are all one. We will sometimes write $\mathbf{1}$ if the size of the matrix is implied by context. Finally, when the vector of all ones is known to be a row vector, we emphasize this fact by denoting the vector as $\mathbf{1}^{\top}$, where the number of columns is implied by context.

Let $A=\left(a_{i j}\right)$ and $\tilde{A}=\left(\tilde{a}_{i j}\right)$ be two $n \times m$ matrices which depend on a real parameter $\omega$, such that the entries of $\tilde{A}$ are always nonnegative. We write $A=O(\tilde{A})$ for $\omega \rightarrow \infty$ if it is true componentwise: $a_{i j}=$ $O\left(\tilde{a}_{i j}\right)$. Thus $A=O(\mathbf{1})$ means that all the components of $A$ are bounded for increasing $\omega$. Multiplication works as expected: if $A=O(\tilde{A})$ and $B=O(\tilde{B})$ then $A B=O(\tilde{A} \tilde{B})$. Note, however, that $O(\mathbf{1} \tilde{A})$ is not equivalent to $O(\tilde{A})$. If $\boldsymbol{f}$ is an $n$-dimensional vector, then $\|\boldsymbol{f}\|$ is of the same asymptotic order as $\tilde{\boldsymbol{f}}^{\top} \mathbf{1}_{n \times 1}=\mathbf{1}^{\top} \tilde{\boldsymbol{f}}$. Furthermore, if $A=O(\mathbf{1})$ is a square matrix, then $\operatorname{det} A=O(1)$. Finally, it can easily be seen that $\|A\|$ and $\left\|A^{\top}\right\|$ have the same asymptotic order.

We can find the asymptotic behaviour of $A^{-1}$ under certain assumptions, which will be used for the proof of Theorem 4.1. An $n \times n$ matrix $A$ satisfies the right-hand regularity condition for a nonsingular matrix $W$ depending on $\omega$ if it can be written as $A=P+G W$, where $G$ is a nonsingular matrix such that $G^{-1}=O(\mathbf{1})$ and $P$ is a matrix such that $P W^{-1}=o(\mathbf{1})$. Likewise, $A$ satisfies the left-hand regularity condition for $W$ if it can be written as $A=P+W G$, where again $G^{-1}=O(\mathbf{1})$, but now $P$ is a matrix such that $W^{-1} P=o(\mathbf{1})$. We can often choose $G$ so that it is independent of $\omega$, in which case it is only necessary to show that $G$ is nonsingular.

Theorem 2.1 If $A$ satisfies the right-hand regularity condition, then $A^{-1}=O\left(W^{-1} \mathbf{1}\right)$. If $A$ satisfies the left-hand regularity condition, then $A^{-1}=O\left(1 W^{-1}\right)$.

Proof: We begin with the case where $A$ satisfies the right-hand regularity condition. Note that $A=$ $\left(P W^{-1} G^{-1}+I\right) G W=(I-M) G W$ for $M=-P W^{-1} G^{-1}$. Since $G^{-1}=O(\mathbf{1})$, it follows that $M=o(\mathbf{1})$ and large $\omega$ ensures that $\|M\|<1$. We thus know that the inverse of $I-M$ exists, and furthermore

$$
(I-M)^{-1}=I+M(I-M)^{-1}=I+o(\mathbf{1})(I-M)^{-1} .
$$

If $(I-M)^{-1}$ was not $O(\mathbf{1})$, we would obtain a contradiction, since the right-hand side of the equality could not be of the same asymptotic order. It follows that $(I-M)^{-1}=O(\mathbf{1})$, and we can write

$$
A^{-1}=W^{-1} G^{-1}(I-M)^{-1}=W^{-1} O(\mathbf{1})=O\left(W^{-1} \mathbf{1}\right) .
$$

We can do something similar for the left-hand regularity condition. Now $A=W G\left(G^{-1} W^{-1} P+I\right)=$ $W G(I-M)$, for $M=-G^{-1} W^{-1} P$. By the same logic as before, the inverse of $I-M$ exists and is $O(\mathbf{1})$. Thus we can write

$$
A^{-1}=(I-M)^{-1} G^{-1} W^{-1}=O\left(1 W^{-1}\right) .
$$

Q.E.D.

We now turn our attention to functions which depend on $\omega$ as a parameter, for example $f(x)=\operatorname{Ai}(-\omega x)$. Let $f$ be such a function, and $\tilde{f}$ a nonnegative constant that depends on $\omega$. We write $f=\mathcal{O}(\tilde{f})$ if the norm of $f$ and its derivatives are all of order $O(\tilde{f})$ as $\omega \rightarrow \infty$. In other words, $\left\|f^{(m)}\right\|=O(\tilde{f})$, for $m=0,1, \ldots$ The most common usage is $f=\mathcal{O}(1)$, which states that $f$ and its derivatives are bounded in $[a, b]$ for increasing $\omega$. We also use this notation for vector-valued and matrix-valued functions in a componentwise manner. Let $A(x)=\left(a_{i j}(x)\right)$ be an $n \times m$ matrix-valued function that depends on $\omega$, and $\tilde{A}=\left(\tilde{a}_{i j}\right)$ an $n \times m$ matrix with nonnegative components, which also depends on $\omega$. We write $A=\mathcal{O}(\tilde{A})$ if it is true componentwise: $a_{i j}=\mathcal{O}\left(\tilde{a}_{i j}\right)$ for $\omega \rightarrow \infty$. 
Note that this class of functions has the following properties, where $A=\mathcal{O}(\tilde{A})$ and $B=\mathcal{O}(\tilde{B})$ are compatible matrix-valued functions, $c=O(\tilde{c})$ is a constant depending on $\omega, m$ is a nonnegative integer and $a \leq x \leq b$ :

$$
\begin{array}{ll}
A(x)=O(\tilde{A}), & A^{(m)}=\mathcal{O}(\tilde{A}), \quad A+B=\mathcal{O}(\tilde{A}+\tilde{B})=\mathcal{O}\left(\max \left\{\tilde{a}_{i j}, \tilde{b}_{i j}\right\}\right), \\
A B=\mathcal{O}(\tilde{A} \tilde{B}), & c A=\mathcal{O}(\tilde{c} \tilde{A}) .
\end{array}
$$

Finally, if $A(x)=O(\tilde{A})$ for all $a \leq x \leq b$, then $\int_{a}^{b} A(x) \mathrm{d} x=O(\tilde{A})$.

\section{Asymptotic expansion}

An asymptotic expansion is a valuable tool in the analysis of integrals, and for large $\omega$ will provide a fairly accurate numerical approximation to $I[\boldsymbol{f}]$. Consider for a moment the one-dimensional oscillator $y=\mathrm{e}^{\mathrm{i} \omega g}$. In the derivation of its asymptotic expansion [3], the fact that $y$ satisfies the differential equation

$$
y^{\prime}(x)=\mathrm{i} \omega g^{\prime}(x) y(x)=A(x) y(x)
$$

was used. The asymptotic expansion follows from writing $y$ as $A^{-1} y^{\prime}$, assuming that $A(x) \neq 0$ in the interval of integration, and integrating by parts:

$$
\int_{a}^{b} f y \mathrm{~d} x=\int_{a}^{b} f A^{-1} y^{\prime} \mathrm{d} x=\left[f A^{-1} y\right]_{a}^{b}-\int_{a}^{b}\left(f A^{-1}\right)^{\prime} y \mathrm{~d} x=\frac{1}{\mathrm{i} \omega}\left[\left(\frac{f}{g^{\prime}}\right)^{\prime} y\right]_{a}^{b}-\frac{1}{\mathrm{i} \omega} \int_{a}^{b}\left(\frac{f}{g^{\prime}}\right)^{\prime} y \mathrm{~d} x .
$$

As $\omega$ becomes large, the error resulting from the integral term decays. We can do something similar for the vector-valued case:

Theorem 3.1 Suppose that $\boldsymbol{y}$ satisfies the differential equation

$$
\boldsymbol{y}^{\prime}(x)=A(x) \boldsymbol{y}(x)
$$

in the interval $[a, b]$, for some invertible matrix-valued function $A$ such that $A^{-1}=\mathcal{O}(\hat{A})$, for $\omega \rightarrow \infty$.

Define

$$
Q_{s}^{A}[\boldsymbol{f}]=\sum_{k=0}^{s-1}(-1)^{k}\left[\boldsymbol{\sigma}_{k}(b)^{\top} A^{-1}(b) \boldsymbol{y}(b)-\boldsymbol{\sigma}_{k}(a)^{\top} A^{-1}(a) \boldsymbol{y}(a)\right],
$$

where

$$
\boldsymbol{\sigma}_{0} \equiv \boldsymbol{f}, \quad \boldsymbol{\sigma}_{k+1}^{\top}=\left(\boldsymbol{\sigma}_{k}^{\top} A^{-1}\right)^{\prime}, \quad k=0,1, \ldots
$$

If $\boldsymbol{f}=\mathcal{O}(\tilde{\boldsymbol{f}})$ and $\boldsymbol{y}(x)=O(\tilde{\boldsymbol{y}})$ for $a \leq x \leq b$, then

$$
Q_{s}^{A}[\boldsymbol{f}]-I[\boldsymbol{f}]=(-1)^{s+1} \int_{a}^{b} \boldsymbol{\sigma}_{s}^{\top} \boldsymbol{y} \mathrm{d} x=O\left(\tilde{\boldsymbol{f}}^{\top} \hat{A}^{s+1} \tilde{\boldsymbol{y}}\right), \quad \omega \rightarrow \infty .
$$

Proof: Note that

$$
\int_{a}^{b} \boldsymbol{\sigma}_{k}^{\top} \boldsymbol{y} \mathrm{d} x=\int_{a}^{b} \boldsymbol{\sigma}_{k}^{\top} A^{-1} \boldsymbol{y}^{\prime} \mathrm{d} x=\left[\boldsymbol{\sigma}_{k}^{\top} A^{-1} \boldsymbol{y}\right]_{a}^{b}-\int_{a}^{b}\left(\boldsymbol{\sigma}_{k}^{\top} A^{-1}\right)^{\prime} \boldsymbol{y} \mathrm{d} x=\left[\boldsymbol{\sigma}_{k}^{\top} A^{-1} \boldsymbol{y}\right]_{a}^{b}-\int_{a}^{b} \boldsymbol{\sigma}_{k+1}^{\top} \boldsymbol{y} \mathrm{d} x .
$$

Thus, by induction, the first equality holds. We now show that $\boldsymbol{\sigma}_{k}^{\top}=\mathcal{O}\left(\tilde{\boldsymbol{f}}^{\top} \hat{A}^{k}\right)$. The case of $k=0$ follows by definition. Otherwise, assume it is true for $k$, and we will prove it for $k+1$ :

$$
\boldsymbol{\sigma}_{k+1}^{\top}=\boldsymbol{\sigma}_{k}^{\top \prime} A^{-1}+\boldsymbol{\sigma}_{k}^{\top} A^{-1^{\prime}}=\mathcal{O}\left(\tilde{\boldsymbol{f}}^{\top} \hat{A}^{k}\right) \mathcal{O}(\hat{A})+\mathcal{O}\left(\tilde{\boldsymbol{f}}^{\top} \hat{A}^{k}\right) \mathcal{O}(\hat{A})=\mathcal{O}\left(\tilde{\boldsymbol{f}}^{\top} \hat{A}^{k+1}\right) .
$$

The theorem now follows since

$$
\int_{a}^{b} \boldsymbol{\sigma}_{s}^{\top} \boldsymbol{y} \mathrm{d} x=\left[\boldsymbol{\sigma}_{s}^{\top} A^{-1} \boldsymbol{y}\right]_{a}^{b}-\int_{a}^{b} \boldsymbol{\sigma}_{s+1}^{\top} \boldsymbol{y} \mathrm{d} x=O\left(\tilde{\boldsymbol{f}}^{\top} \hat{A}^{s+1} \tilde{\boldsymbol{y}}\right)+O\left(\tilde{\boldsymbol{f}}^{\top} \hat{A}^{s+1} \tilde{\boldsymbol{y}}\right)=O\left(\tilde{\boldsymbol{f}}^{\top} \hat{A}^{s+1} \tilde{\boldsymbol{y}}\right) .
$$



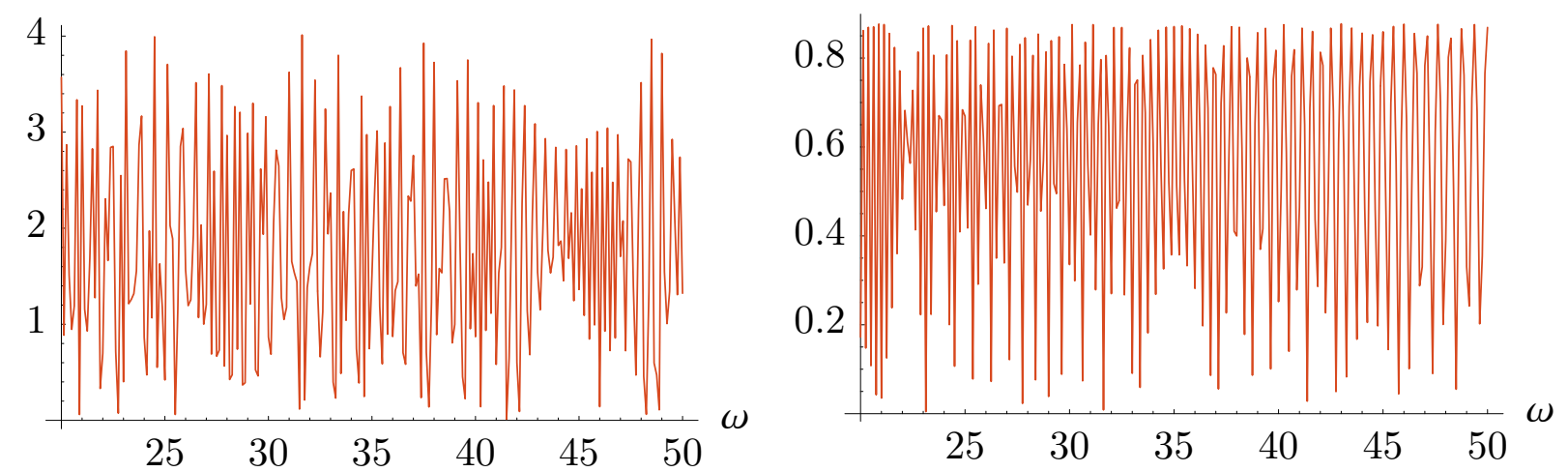

Figure 1: The error of $Q_{1}^{A}[\boldsymbol{f}]$ scaled by $\omega^{7 / 4}$ (left figure), compared to the error of $Q_{2}^{A}[\boldsymbol{f}]$ scaled by $\omega^{13 / 4}$ (right figure), for $I[\boldsymbol{f}]=\int_{1}^{2}\left[\cos x \mathrm{Ai}(-\omega x)-\omega \mathrm{e}^{x} \mathrm{Ai}^{\prime}(-\omega x)\right] \mathrm{d} x$.

Corollary 3.2 follows from Theorem 3.1, and will be used in the proof of Theorem 4.1.

Corollary 3.2 Suppose that

$$
\boldsymbol{f}(a)=\boldsymbol{f}(b)=\boldsymbol{f}^{\prime}(a)=\boldsymbol{f}^{\prime}(b)=\cdots=\boldsymbol{f}^{(s-1)}(a)=\boldsymbol{f}^{(s-1)}(b)=\mathbf{0} .
$$

Then

$$
I[\boldsymbol{f}]=O\left(\tilde{\boldsymbol{f}}^{\top} \hat{A}^{s+1} \tilde{\boldsymbol{y}}\right) .
$$

The asymptotic expansion for $y(x)=\mathrm{e}^{\mathrm{i} \omega g(x)}$ follows immediately, assuming that $g^{\prime} \neq 0$, in which case $A^{-1}(x)=1 /(\mathrm{i} \omega g(x))=O\left(\omega^{-1}\right)$. Thus $Q_{s}^{A}[f]$ approximates $I[f]$ with an order $O\left(\omega^{-s-1}\right)$. For the two-dimensional case, examples include, assuming $0<a<b$,

$$
\begin{aligned}
& \boldsymbol{y}(x)=\left(\begin{array}{c}
J_{m-1}(\omega x) \\
J_{m}(\omega x)
\end{array}\right)=O\left(\omega^{-1 / 2} \mathbf{1}\right), \quad A^{-1}=\mathcal{O}\left(\begin{array}{cc}
\omega^{-2} & \omega^{-1} \\
\omega^{-1} & \omega^{-2}
\end{array}\right)=\mathcal{O}\left(\omega^{-1} \mathbf{1}\right), \\
& \boldsymbol{y}(x)=\left(\begin{array}{c}
\operatorname{Ai}(-\omega x) \\
-\omega \mathrm{Ai}^{\prime}(-\omega x)
\end{array}\right)=O\left(\begin{array}{c}
\omega^{-1 / 4} \\
\omega^{5 / 4}
\end{array}\right), \quad A^{-1}=\mathcal{O}\left(\begin{array}{cc}
0 & \omega^{-3} \\
1 & 0
\end{array}\right),
\end{aligned}
$$

where the asymptotics of the Bessel and Airy functions can be found in [1]. In the Bessel case, each component of $A^{-1}$ is $\mathcal{O}\left(\omega^{-1}\right)$, hence, assuming that $\boldsymbol{f}=\mathcal{O}(\mathbf{1})$, we have an error of order

$$
\tilde{\boldsymbol{f}}^{\top} \hat{A}^{s+1} \tilde{\boldsymbol{y}}=O\left(\left\|\hat{A}^{s+1}\right\|\|\tilde{\boldsymbol{y}}\|\right)=O\left(\omega^{-s-\frac{3}{2}}\right) .
$$

In the Airy case, we know that

$$
\hat{A}^{2 k} \tilde{\boldsymbol{y}}=\left(\begin{array}{cc}
\omega^{-3 k} & 0 \\
0 & \omega^{-3 k}
\end{array}\right) \tilde{\boldsymbol{y}}=\left(\begin{array}{c}
\omega^{-3 k-1 / 4} \\
\omega^{-3 k+5 / 4}
\end{array}\right), \quad \hat{A}^{2 k+1} \tilde{\boldsymbol{y}}=\left(\begin{array}{cc}
0 & \omega^{-3(k+1)} \\
\omega^{-3 k} & 0
\end{array}\right) \tilde{\boldsymbol{y}}=\left(\begin{array}{c}
\omega^{-3 k-7 / 4} \\
\omega^{-3 k-1 / 4}
\end{array}\right) .
$$

Thus, if $\tilde{f}=\mathbf{1}$,

$$
\tilde{\boldsymbol{f}}^{\top} \hat{A}^{s+1} \tilde{\boldsymbol{y}}=O\left(\omega^{-\frac{3}{2} s-\frac{1}{4}}\right) .
$$

On the other hand, if $\tilde{\boldsymbol{f}}=(1,0)^{\top}$, then

$$
\tilde{\boldsymbol{f}}^{\top} \hat{A}^{s+1} \tilde{\boldsymbol{y}}=O\left(\omega^{-\frac{3}{2} s-\frac{7}{4}}\right) .
$$

As a simple example, consider the integral

$$
\int_{1}^{2} \boldsymbol{f}^{\top} \boldsymbol{y} \mathrm{d} x=\int_{1}^{2}\left[\cos x \operatorname{Ai}(-\omega x)-\omega \mathrm{e}^{x} \mathrm{Ai}^{\prime}(-\omega x)\right] \mathrm{d} x,
$$

for $\boldsymbol{f}(x)=\left(\cos x, \mathrm{e}^{x}\right)^{\top}$ and $\boldsymbol{y}(x)=\left(\mathrm{Ai}(-\omega x),-\omega \mathrm{Ai}^{\prime}(-\omega x)\right)^{\top}$. Figure 1 compares the one-term and twoterm expansions. As can be seen, adding an additional term does indeed increase the asymptotic order by $3 / 2$. 


\section{Levin-type methods}

The fundamental problem with using an asymptotic expansion as a numerical approximation is that for fixed $\omega$ the accuracy is limited: the infinite sum does not necessarily converge. To combat this issue, we will derive a Levin-type method that has the same asymptotic behaviour as the asymptotic expansion, whilst providing the ability to decrease error further. In [5], a method was developed to compute integrals using a collocation system. The current author generalized this method to include multiplicities in [7], for the specific oscillator $\mathrm{e}^{\mathrm{i} \omega g}$. By adding multiplicities to the endpoints, we obtain a method with higher asymptotic order. In this section, we complete the generalization for vector-valued kernels. We will use the asymptotic expansion to determine the asymptotic order of the Levin-type method. Note that we include cases that were not analysed in [5], such as the Airy function case. When the Levin-type method is equivalent to the original method, we obtain the asymptotic bound derived in $[\mathbf{1 0}]$, which is more accurate than the bound found in $[\mathbf{5}]$.

Had we known a vector-valued function $\boldsymbol{F}$ such that

$$
\left(\boldsymbol{F}^{\top} \boldsymbol{y}\right)^{\prime}=\boldsymbol{f}^{\top} \boldsymbol{y}
$$

then computing the integral $I[\boldsymbol{f}]$ would have been trivial: $I[\boldsymbol{f}]=\left[\boldsymbol{F}^{\top} \boldsymbol{y}\right]_{a}^{b}$. We can rewrite this condition as

$$
\mathcal{L}[\boldsymbol{F}]=\boldsymbol{f}, \quad \text { for } \quad \mathcal{L}[\boldsymbol{F}]=\boldsymbol{F}^{\prime}+A^{\top} \boldsymbol{F} .
$$

Finding $\boldsymbol{F}$ explicitly is in general not possible. However, we can approximate this function using collocation. Let $\boldsymbol{v}(x)=\sum_{k=1}^{n} c_{k} \boldsymbol{\psi}_{k}(x)$ for some set of basis functions $\left\{\boldsymbol{\psi}_{k}\right\}$, where $\boldsymbol{\psi}_{k}: \mathbb{R} \rightarrow \mathbb{R}^{d}$, and $n=d \sum m_{k}$, i.e., the total number of equations in the system (4.1). For a sequence of nodes $\left\{x_{1}, \ldots, x_{\nu}\right\}$ and multiplicities $\left\{m_{1}, \ldots, m_{\nu}\right\}$, we determine the coefficients of $\boldsymbol{v}$ by solving the system

$$
\mathcal{L}[\boldsymbol{v}]\left(x_{k}\right)=\boldsymbol{f}\left(x_{k}\right), \ldots, \mathcal{L}[\boldsymbol{v}]^{\left(m_{k}-1\right)}\left(x_{k}\right)=\boldsymbol{f}^{\left(m_{k}-1\right)}\left(x_{k}\right), \quad k=1,2, \ldots, \nu .
$$

We then define a Levin-type method as

$$
Q^{L}[\boldsymbol{f}]=\boldsymbol{v}(b)^{\top} \boldsymbol{y}(b)-\boldsymbol{v}(a)^{\top} \boldsymbol{y}(a) .
$$

Let $\mathcal{P}[\boldsymbol{g}]$ be the vector consisting of the function $\boldsymbol{g}$ evaluated at each node and multiplicity, written in partitioned form as

$$
\mathcal{P}[\boldsymbol{g}]=\left(\begin{array}{c}
\boldsymbol{g}\left(x_{1}\right) \\
\vdots \\
\boldsymbol{g}^{\left(m_{1}-1\right)}\left(x_{1}\right) \\
\vdots \\
\boldsymbol{g}\left(x_{\nu}\right) \\
\vdots \\
\boldsymbol{g}^{\left(m_{\nu}-1\right)}\left(x_{\nu}\right)
\end{array}\right) .
$$

Furthermore, let $\Psi=\mathcal{O}(\tilde{\Psi})$ be the $d \times n$ matrix-valued function whose $k$ th column equals $\boldsymbol{\psi}_{k}$, written in partitioned form as

$$
\Psi(x)=\left(\boldsymbol{\psi}_{1}(x), \ldots, \boldsymbol{\psi}_{n}(x)\right) .
$$

Then we can write the system (4.1) as $B \boldsymbol{c}=\boldsymbol{\varphi}$, where $\boldsymbol{c}=\left(c_{1}, \ldots, c_{n}\right)^{\top}$,

$$
B=\mathcal{P}[\mathcal{L}[\Psi]]=\left(\mathcal{P}\left[\mathcal{L}\left[\boldsymbol{\psi}_{1}\right]\right], \ldots, \mathcal{P}\left[\mathcal{L}\left[\boldsymbol{\psi}_{n}\right]\right]\right), \quad \boldsymbol{\varphi}=\mathcal{P}[\boldsymbol{f}]=O(\tilde{\boldsymbol{\varphi}})=O\left(\begin{array}{c}
\tilde{\boldsymbol{f}} \\
\vdots \\
\tilde{\boldsymbol{f}}
\end{array}\right)
$$

Thus $\boldsymbol{v}=\Psi \boldsymbol{c}$. 
Theorem 4.1 Assume that $\boldsymbol{f}=\mathcal{O}(\tilde{\boldsymbol{f}}), A=\mathcal{O}(\tilde{A})$ and $A^{-1}=\mathcal{O}(\hat{A})$, where $\tilde{A} \hat{A}=O(\mathbf{1})$. Let $s=$ $\min \left\{m_{1}, m_{\nu}\right\}$. If $B$ satisfies the right-hand regularity condition for $W$ such that $\left(I+\tilde{A}^{\top}\right) \tilde{\Psi} W^{-1}=O\left(\mathbf{1}_{d \times n}\right)$, then

$$
Q^{L}[\boldsymbol{f}]-I[\boldsymbol{f}]=O\left(\tilde{\boldsymbol{f}}^{\top} \mathbf{1} \hat{A}^{s+1} \tilde{\boldsymbol{y}}\right) .
$$

If $B$ satisfies the left-hand regularity condition, then

$$
Q^{L}[\boldsymbol{f}]-I[\boldsymbol{f}]=O\left(\tilde{\boldsymbol{\varphi}}^{\top} W^{-1} \mathbf{1} \hat{A}^{s} \tilde{\boldsymbol{y}}\right),
$$

subject to the assumption that $\Psi=\mathcal{O}(\mathbf{1})$ and $\hat{A}^{k+1} \tilde{\boldsymbol{y}}=O\left(\hat{A}^{k} \tilde{\boldsymbol{y}}\right)$.

Proof: We begin with the right-hand regularity condition case. Note that

$$
Q^{L}[\boldsymbol{f}]-I[\boldsymbol{f}]=I[\mathcal{L}[\boldsymbol{v}]-\boldsymbol{f}] .
$$

If we show that $\mathcal{L}[\boldsymbol{v}]=\mathcal{O}(\mathbf{1} \tilde{\boldsymbol{f}})$ then Corollary 3.2 would imply

$$
I[\mathcal{L}[\boldsymbol{v}]-\boldsymbol{f}]=O\left(\tilde{\boldsymbol{f}}^{\top} \mathbf{1} \hat{A}^{s+1} \tilde{\boldsymbol{y}}\right) .
$$

Note that $\boldsymbol{c}=B^{-1} \boldsymbol{\varphi}=O\left(W^{-1} \mathbf{1}_{n \times n} \tilde{\boldsymbol{\varphi}}\right)$. Thus

$$
\mathcal{L}[\boldsymbol{v}]=\mathcal{L}[\Psi \boldsymbol{c}]=\left(\Psi^{\prime}+A^{\top} \Psi\right) \boldsymbol{c}=\mathcal{O}\left(\left(I+\tilde{A}^{\top}\right) \tilde{\Psi} W^{-1} \mathbf{1}_{n \times n} \tilde{\boldsymbol{\varphi}}\right)=\mathcal{O}\left(\mathbf{1}_{d \times n} \tilde{\boldsymbol{\varphi}}\right) .
$$

But $\mathbf{1}_{d \times n} \tilde{\boldsymbol{\varphi}}$ is a vector with $d$ entries, each of order $\mathbf{1}^{\top} \tilde{\boldsymbol{f}}$. Hence $\mathcal{L}[\boldsymbol{v}]=\mathcal{O}\left(\mathbf{1}_{d \times d} \tilde{\boldsymbol{f}}\right)$, and the theorem follows from Corollary 3.2.

Now consider the left-hand regularity condition case, where $\boldsymbol{c}=B^{-1} \boldsymbol{\varphi}=O\left(\mathbf{1} W^{-1} \tilde{\boldsymbol{\varphi}}\right)$. Thus

$$
\mathcal{L}[\boldsymbol{v}]=\mathcal{L}[\Psi \boldsymbol{c}]=\left(\Psi^{\prime}+A^{\top} \Psi\right) \boldsymbol{c}=\mathcal{O}\left(\left(I+\tilde{A}^{\top}\right) \tilde{\Psi} 1 W^{-1} \tilde{\boldsymbol{\varphi}}\right) .
$$

We know that $(I+\tilde{A}) \hat{A}^{s+1} \tilde{\boldsymbol{y}}=\left(\hat{A}^{s+1}+\hat{A}^{s}\right) \tilde{\boldsymbol{y}}=O\left(\hat{A}^{s} \tilde{\boldsymbol{y}}\right)$. Thus the theorem follows.

The following corollary shows, under fairly general conditions, that a polynomial basis will always obtain the desired order of error in a Levin-type method.

Corollary 4.2 Suppose that

$$
\Psi(x)=\left(I, x I, \ldots, x^{n / d} I\right)
$$

is the standard polynomial basis and $A(x)=D K(x) D^{-1}$ for some $K$ such that $\hat{K}=o(\mathbf{1})$. Then a Levin-type method has an order of error

$$
Q^{L}[\boldsymbol{f}]-I[\boldsymbol{f}]=O\left(\tilde{\boldsymbol{f}}^{\top} D^{-1} \hat{K} \mathbf{1} \hat{K}^{s} \tilde{\boldsymbol{y}}\right) .
$$

Proof: We first consider the case where all multiplicities equal one. The first half of this proof is based loosely on a similar proof in [10], put into the framework of this paper and generalized to include nonzero multiplicities. We begin with the case where $D=I$, hence $A=K$. We will show, with a specified choice of $W$, that the left-hand regularity condition is always satisfied. We can write

$$
\begin{gathered}
B=P+W G, \quad P=\mathcal{P}\left[\Psi^{\prime}\right]=\left(\begin{array}{cccc}
O & I & \cdots & (\nu-1) x_{1}^{\nu-2} I \\
\vdots & \vdots & \ddots & \vdots \\
O & I & \cdots & (\nu-1) x_{\nu}^{\nu-2} I
\end{array}\right) \\
W=\left(\begin{array}{ccc}
K^{\top}\left(x_{1}\right) & & \\
& \ddots & \\
& & K^{\top}\left(x_{\nu}\right)
\end{array}\right), \quad G=\mathcal{P}[\Psi]=\left(\begin{array}{ccc}
I & \cdots & x_{1}^{\nu-1} I \\
\vdots & \ddots & \vdots \\
I & \cdots & x_{\nu}^{\nu-1} I
\end{array}\right) .
\end{gathered}
$$



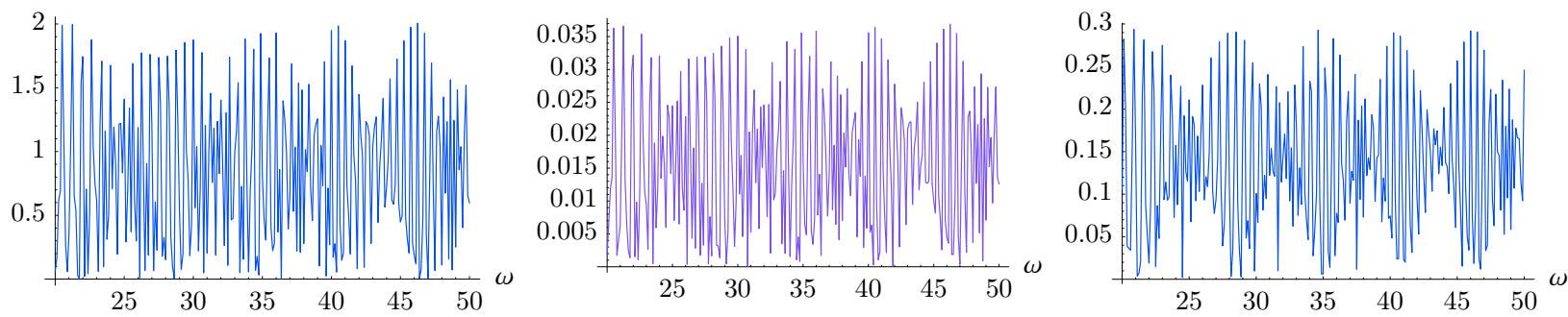

Figure 2: The error scaled by $\omega^{7 / 4}$ of $Q^{L}[\boldsymbol{f}]$ with endpoints for nodes and multiplicities both one (left figure), $Q^{L}[\boldsymbol{f}]$ with nodes $(1,4 / 3,5 / 3,2)$ and multiplicities all one (middle figure), and the error scaled by $\omega^{13 / 4}$ of $Q^{L}[\boldsymbol{f}]$ with endpoints for nodes and multiplicities both two (right figure), for $I[\boldsymbol{f}]=\int_{1}^{2} \cos x \mathrm{Ai}(-\omega x)-$ $\omega \mathrm{e}^{x} \mathrm{Ai}^{\prime}(-\omega x) \mathrm{d} x$.

Note that $G$ is a block Vandermonde matrix, hence nonsingular. It is also independent of $\omega$, hence $G^{-1}=$ $O(\mathbf{1})$. Furthermore $W$ is nonsingular, and

$$
W^{-1}=\mathcal{O}\left(\begin{array}{lll}
\hat{K}^{\top} & & \\
& \ddots & \\
& & \hat{K}^{\top}
\end{array}\right)
$$

Thus $W^{-1} P=o(\mathbf{1})$, since $P=O(\mathbf{1})$ and $W^{-1}=o(\mathbf{1})$. But $\tilde{\varphi}^{\top} W^{-1} \mathbf{1}=O\left(\tilde{\boldsymbol{f}}^{\top} \hat{K}^{\top} \mathbf{1}\right)$, and the order of error follows from Theorem 4.1.

We now handle the case where $D \neq I$. Note that $\boldsymbol{y}_{2}=D \boldsymbol{y}$ satisfies the differential equation $\boldsymbol{y}_{2}^{\prime}=K \boldsymbol{y}_{2}$. Furthermore

$$
I[\boldsymbol{f}]=\int_{a}^{b} \boldsymbol{f}^{\top} \boldsymbol{y} \mathrm{d} x=\int_{a}^{b} \boldsymbol{f}^{\top} D^{-1} \boldsymbol{y}_{2} \mathrm{~d} x=\int_{a}^{b} \boldsymbol{f}_{2}^{\top} \boldsymbol{y}_{2} \mathrm{~d} x,
$$

for $\boldsymbol{f}_{2}=D^{-1} \boldsymbol{f}$. From the first half of this proof, we know that a Levin-type method for oscillator $\boldsymbol{y}_{2}$ has the requisite order of error. We now show that this new Levin-type method is equivalent to the original Levin-type method. Let $\boldsymbol{v}_{2}$ be the collocation function from the Levin-type method with $\boldsymbol{y}_{2}$. Then we wish to show that

$$
\boldsymbol{v}^{\top} \boldsymbol{y}=\boldsymbol{v}_{2}^{\top} \boldsymbol{y}_{2}=\boldsymbol{v}_{2}^{\top} D \boldsymbol{y}
$$

Let

$$
\bar{D}=\left(\begin{array}{ccc}
D & & \\
& \ddots & \\
& & D
\end{array}\right)
$$

It can easily be seen that $P$ and $G$ commute with $\bar{D}$. Thus

$$
\bar{D}\left(P+\bar{D}^{-1} W \bar{D} G\right) \bar{D}^{-1}=P+W G,
$$

It follows that, where $B_{2}=P+\bar{D}^{-1} W \bar{D} G$ is the matrix associated with the new Levin-type method,

$$
D \boldsymbol{v}_{2}=D \Psi B_{2}^{-1} \varphi_{2}=\Psi \bar{D}\left(P+\bar{D}^{-1} W \bar{D} G\right)^{-1} \bar{D}^{-1} \varphi=\Psi(P+W G)^{-1} \varphi=\Psi B^{-1} \varphi=\boldsymbol{v} .
$$

The case of multiplicities is handled by a trivial limiting argument.

Q.E.D.

The Bessel case satisfies the conditions of this corollary with $D=I$. For the Airy case, we take $D=\operatorname{diag}\left(\omega^{3 / 2}, 1\right)$, in which case

$$
K(x)=D^{-1} A(x) D=\left(\begin{array}{cc}
0 & \omega^{3 / 2} \\
-\omega^{3 / 2} x & 0
\end{array}\right)
$$

does indeed satisfy the requisite property. The asymptotic order of the error predicted by the preceding corollary is equivalent to that of the asymptotic expansion for both the case where $\tilde{\boldsymbol{f}}=\mathbf{1}$ and $\tilde{\boldsymbol{f}}=(1,0)^{\top}$. 

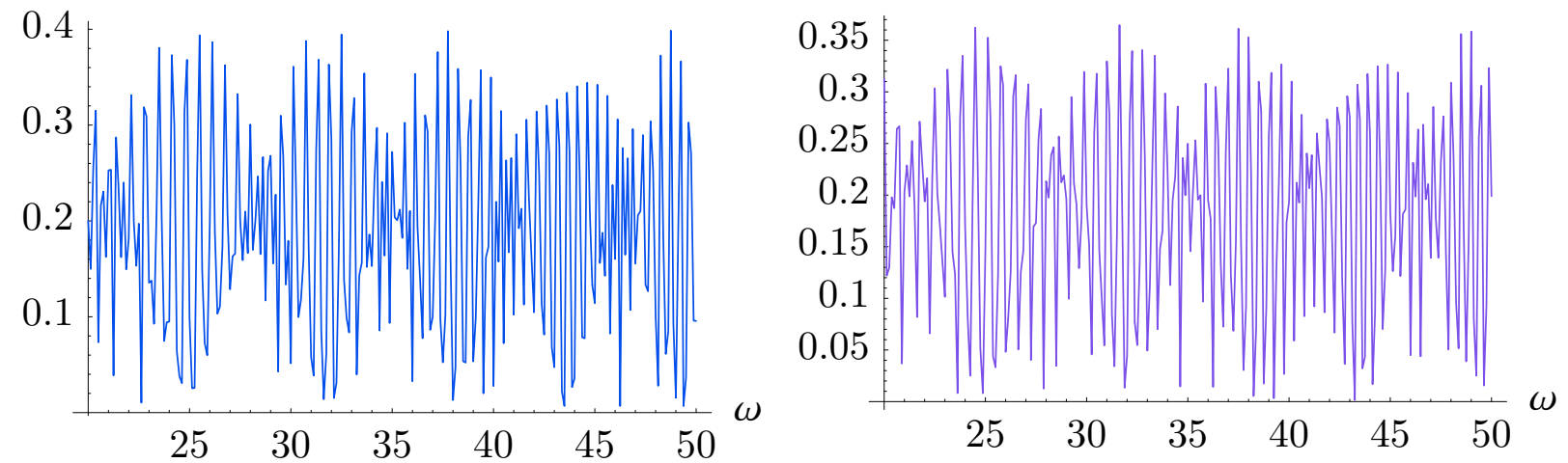

Figure 3: The error scaled by $\omega^{13 / 4}$ of $Q^{L}[\boldsymbol{f}]$ with endpoints for nodes and multiplicities both one (left figure), compared to the error scaled by $\omega^{19 / 4}$ of $Q^{L}[\boldsymbol{f}]$ with endpoints for nodes and multiplicities both two (right figure), for $I[\boldsymbol{f}]=\int_{1}^{2} \mathrm{Ai}(-\omega x) \mathrm{d} x$.

Returning to the example of Figure 1, we now approximate the same integral using a Levin-type method in Figure 2. Using exactly the same information as the asymptotic expansion, we reduce the error by a factor of two. Unlike in the case of an asymptotic expansion, we also have the ability to reduce the error by adding nodes in the interior of the interval. Adding just two nodes, one at $4 / 3$ and one at $5 / 3$, reduces the error by a factor of 100 . This figure also demonstrates that adding multiplicities to the endpoints does indeed increase the asymptotic order.

As another example, consider the computation of the first moment of the Airy function Ai, in particular $\int_{1}^{2} \mathrm{Ai}(-\omega x) \mathrm{d} x$. In this case, $\boldsymbol{y}$ remains the same, while we take $\boldsymbol{f}=(1,0)^{\top}$. As predicted, Figure 3 shows that the approximation has an error of order $\omega^{13 / 4}$ with multiplicities both one, which increases to $\omega^{19 / 4}$ with the addition of multiplicities. Note that this is indeed a higher asymptotic order than the previous Airy case.

Remark: With this approximation in hand we can immediately approximate any of the higher moments, using the integral relation

$$
\int x^{k+3} \operatorname{Ai}(x) \mathrm{d} x=x^{k+2} \operatorname{Ai}^{\prime}(x)-(k+2) x^{k+1} \operatorname{Ai}(x)+(k+1)(k+2) \int x^{k} \mathrm{Ai}(x) \mathrm{d} x,
$$

cf. [1]. This presents the possibility of constructing a Filon-type method [3], where we approximate $\int_{a}^{b} f(x) \mathrm{Ai}(-\omega x) \mathrm{d} x$ by interpolating $f$ by a polynomial $v$, and using the formulæ for the moments to compute $\int_{a}^{b} v(x) \mathrm{Ai}(-\omega x) \mathrm{d} x$. As this idea is tangential to the topic of this paper, we will not investigate it further.

Finally, consider the integral $\int_{1}^{2}\left[x^{-2} J_{2}(\omega x)+x^{3} J_{3}(\omega x)\right] \mathrm{d} x$. In Figure 4 we compare two methods of order $O\left(\omega^{-7 / 2}\right)$ : the first with endpoints for nodes and multiplicities both equal to two, and the second with an additional node at $3 / 2$ with multiplicity one. We obtain the expected order of error and adding an additional interpolation point further decreases the error.

\section{Asymptotic basis}

One key - and easily overlooked - degree of freedom in a Levin-type method is in the choice of basis. Though the obvious choice of using polynomials does indeed provide good approximation, it ignores the wealth of information about $\boldsymbol{f}$ and $A$ which could be used to make $\mathcal{L}[\boldsymbol{v}]$ close to $\boldsymbol{f}$. In $[\boldsymbol{7}]$ it was noted that for the $\mathrm{e}^{\mathrm{i} \omega g}$ oscillator, using the functions $\sigma_{k}$ from the asymptotic expansion as a basis caused the order of the resulting Levin-type method to increase with each additional node point. In this section we show that this carries over to vector-valued kernels as well. This observation is of considerably more importance for the vector-valued case, since it allows us to derive a high-order approximation with a significantly smaller system. Note, however, that we still require the same number of derivatives for $\boldsymbol{f}$ and $A$. 

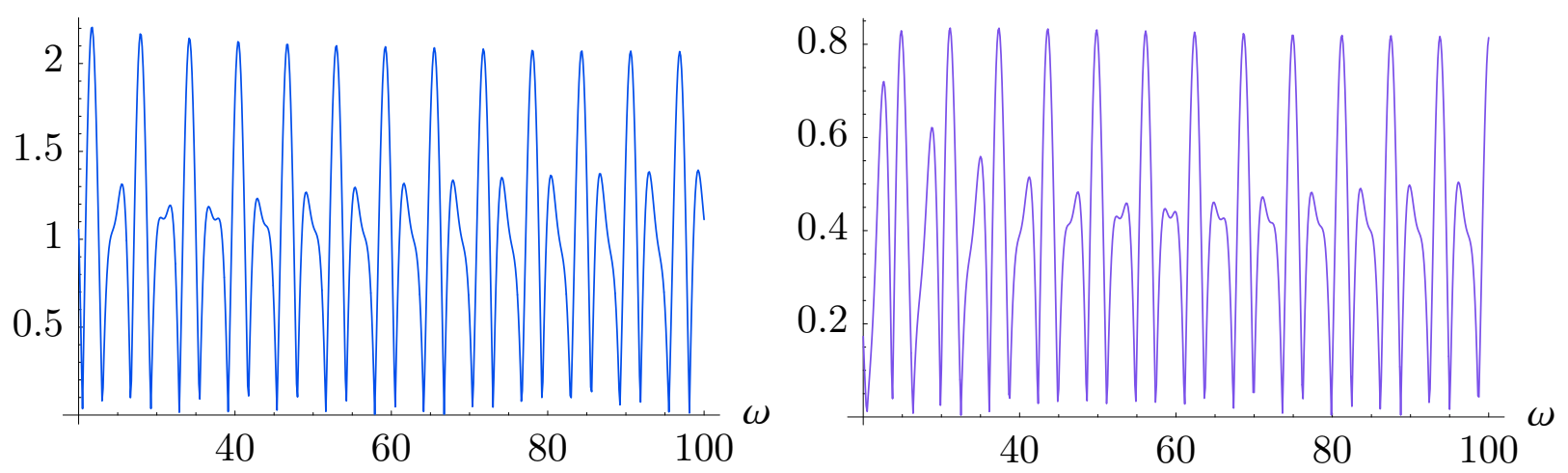

Figure 4: The error scaled by $\omega^{7 / 2}$ of $Q^{L}[\boldsymbol{f}]$ with endpoints for nodes and multiplicities both two (left figure), compared to $Q^{L}[\boldsymbol{f}]$ with nodes $\{1,3 / 2,2\}$ and multiplicities $\{2,1,2\}$ (right figure), for $I[\boldsymbol{f}]=$ $\int_{1}^{2}\left[x^{-2} J_{2}(\omega x)+x^{3} J_{3}(\omega x)\right] \mathrm{d} x$.

Define the asymptotic basis as

$$
\boldsymbol{\psi}_{1}=A^{-\top} \boldsymbol{f}, \quad \boldsymbol{\psi}_{k+1}=A^{-\top} \boldsymbol{\psi}_{k}^{\prime}, \quad k=1,2, \ldots
$$

The following theorem states that under this choice of basis, a Levin-type method will have a higher asymptotic order.

Theorem 5.1 Suppose that $\boldsymbol{f}=\mathcal{O}(\mathbf{1}), \hat{A}^{(k+1) \top} \tilde{\boldsymbol{f}}=O\left(\hat{A}^{k \top} \tilde{\boldsymbol{f}}\right)$, and that $B$ satisfies the right-hand regularity condition for

$$
W=\operatorname{diag}\left(1,\|\hat{A}\|, \ldots,\left\|\hat{A}^{n-1}\right\|\right) .
$$

Then

$$
Q^{B}[\boldsymbol{f}]-I[\boldsymbol{f}]=O\left(\left\|\hat{A}^{n}\right\| \mathbf{1}^{\top} \hat{A}^{s+1} \tilde{\boldsymbol{y}}\right)
$$

where $Q^{B}[\boldsymbol{f}]$ is a Levin-type method using the asymptotic basis.

Proof: $\quad$ Note that $\boldsymbol{\psi}_{k}=\mathcal{O}\left(\hat{A}^{k \top} \tilde{\boldsymbol{f}}\right)$. We find that

$$
\tilde{\Psi}=\left(\hat{A}^{\top} \tilde{\boldsymbol{f}}, \ldots,\left(\hat{A}^{n}\right)^{\top} \tilde{\boldsymbol{f}}\right) \Rightarrow\left(I+\tilde{A}^{\top}\right) \tilde{\Psi} W^{-1}=O\left(\tilde{\boldsymbol{f}}, \frac{\hat{A}^{\top} \tilde{\boldsymbol{f}}}{\|\hat{A}\|}, \ldots, \frac{\left(\hat{A}^{n-1}\right)^{\top} \tilde{\boldsymbol{f}}}{\left\|\hat{A}^{n-1}\right\|}\right)=O(\mathbf{1})
$$

where we used the fact that $\|\hat{A}\|$ and $\left\|\hat{A}^{\top}\right\|$ have the same asymptotic order. Thus the conditions for the first half of Theorem 4.1 are satisfied.

We now show that $\mathcal{L}[\boldsymbol{v}]-\boldsymbol{f}=\mathcal{O}\left(\left\|\hat{A}^{n}\right\| \mathbf{1}\right)$. Note that

$$
\begin{aligned}
\mathcal{L}[\boldsymbol{v}]-\boldsymbol{f} & =\sum_{k=1}^{n} c_{k} \mathcal{L}\left[\boldsymbol{\psi}_{k}\right]-\boldsymbol{f}=\sum_{k=1}^{n} c_{k}\left(\boldsymbol{\psi}_{k}^{\prime}+A^{\top} \boldsymbol{\psi}_{k}\right)-\boldsymbol{f} \\
& =c_{1} A^{\top} \boldsymbol{\psi}_{1}+c_{1} A^{\top} \boldsymbol{\psi}_{2}+\sum_{k=2}^{n} c_{k}\left(A^{\top} \boldsymbol{\psi}_{k+1}+A^{\top} \boldsymbol{\psi}_{k}\right)-A^{\top} \boldsymbol{\psi}_{1} \\
& =A^{\top}\left[\left(c_{1}-1\right) \boldsymbol{\psi}_{1}+\sum_{k=2}^{n}\left(c_{k-1}+c_{k}\right) \boldsymbol{\psi}_{k}+c_{n} \boldsymbol{\psi}_{n+1}\right] \\
& =\frac{A^{\top}}{\operatorname{det} B}\left[\left(\operatorname{det} D_{1}-\operatorname{det} B\right) \boldsymbol{\psi}_{1}+\sum_{k=2}^{n}\left(\operatorname{det} D_{k-1}+\operatorname{det} D_{k}\right) \boldsymbol{\psi}_{k}+\operatorname{det} D_{n} \boldsymbol{\psi}_{n+1}\right]
\end{aligned}
$$


where the matrix $D_{k}$ is the matrix $B$ with its $k$ th column replaced by $\boldsymbol{\varphi}=\mathcal{P}[\boldsymbol{f}]$, cf. Cramer's rule. Because the right-hand regularity condition is satisfied, we determine that

$$
(\operatorname{det} B)^{-1}=\operatorname{det} B^{-1}=\operatorname{det} W^{-1} \operatorname{det} O(\mathbf{1})=\left(\prod_{j=0}^{n-1}\left\|\hat{A}^{j}\right\|\right)^{-1} O(1)=O\left(\prod_{j=0}^{n-1}\left\|\hat{A}^{j}\right\|^{-1}\right) .
$$

We now wish to show that the term multiplied by $\psi_{k}$ in (5.1), namely $\operatorname{det} D_{1}-\operatorname{det} B, \operatorname{det} D_{k-1}+\operatorname{det} D_{k}$ or $\operatorname{det} D_{n}$, is of order $O\left(\prod_{\substack{j=0 \\ j \neq k-1}}^{n}\left\|\hat{A}^{j}\right\|\right)$. Note that

$$
\begin{aligned}
& \operatorname{det} D_{1}-\operatorname{det} B=\operatorname{det}\left(\mathcal{P}\left[A^{\top} \boldsymbol{\psi}_{1}\right], \mathcal{P}\left[A^{\top}\left(\boldsymbol{\psi}_{3}+\boldsymbol{\psi}_{2}\right)\right], \ldots, \mathcal{P}\left[A^{\top}\left(\boldsymbol{\psi}_{n+1}+\boldsymbol{\psi}_{n}\right)\right]\right) \\
&-\operatorname{det}\left(\mathcal{P}\left[A^{\top}\left(\boldsymbol{\psi}_{2}+\boldsymbol{\psi}_{1}\right)\right], \mathcal{P}\left[A^{\top}\left(\boldsymbol{\psi}_{3}+\boldsymbol{\psi}_{2}\right)\right], \ldots, \mathcal{P}\left[A^{\top}\left(\boldsymbol{\psi}_{n+1}+\boldsymbol{\psi}_{n}\right)\right]\right) \\
&=-\operatorname{det}\left(\mathcal{P}\left[A^{\top} \boldsymbol{\psi}_{2}\right], \mathcal{P}\left[A^{\top}\left(\boldsymbol{\psi}_{3}+\boldsymbol{\psi}_{2}\right)\right], \ldots, \mathcal{P}\left[A^{\top}\left(\boldsymbol{\psi}_{n+1}+\boldsymbol{\psi}_{n}\right)\right]\right) \\
&=-\operatorname{det}\left(\mathcal{P}\left[A^{\top} \boldsymbol{\psi}_{2}\right], \mathcal{P}\left[A^{\top} \boldsymbol{\psi}_{3}\right], \ldots, \mathcal{P}\left[A^{\top} \boldsymbol{\psi}_{n+1}\right]\right) \\
&=-\operatorname{det}\left(\mathcal{P}\left[\boldsymbol{\psi}_{1}^{\prime}\right], \mathcal{P}\left[\boldsymbol{\psi}_{2}^{\prime}\right], \ldots, \mathcal{P}\left[\boldsymbol{\psi}_{n}^{\prime}\right]\right) .
\end{aligned}
$$

Since $\mathcal{P}\left[\boldsymbol{\psi}_{k}^{\prime}\right]=O\left(\tilde{\boldsymbol{f}}^{\top} \hat{A}^{k} \mathbf{1}, \ldots, \tilde{\boldsymbol{f}}^{\top} \hat{A}^{k} \mathbf{1}\right)^{\top}=O\left(\left\|\hat{A}^{k}\right\|, \ldots,\left\|\hat{A}^{k}\right\|\right)^{\top}$, the $k$ th column in this determinant is composed of entries of order $O\left(\left\|\hat{A}^{k}\right\|\right)$, thus the determinant is of the requisite order $O\left(\prod_{k=1}^{n}\left\|\hat{A}^{k}\right\|\right)$. Likewise, writing for brevity $\mathcal{P}\left[\mathcal{L}\left[\boldsymbol{\psi}_{k}\right]\right]=\mathcal{P}\left[A^{\top}\left(\boldsymbol{\psi}_{k}+\boldsymbol{\psi}_{k+1}\right)\right]$ as $\boldsymbol{b}_{k}$,

$$
\begin{aligned}
& \operatorname{det} D_{k-1}+\operatorname{det} D_{k}=\operatorname{det}\left(\boldsymbol{b}_{1}, \ldots, \boldsymbol{b}_{k-2}, \mathcal{P}\left[A^{\top} \boldsymbol{\psi}_{1}\right], \boldsymbol{b}_{k}, \boldsymbol{b}_{k+1}, \ldots, \boldsymbol{b}_{n}\right) \\
&+\operatorname{det}\left(\boldsymbol{b}_{1}, \ldots, \boldsymbol{b}_{k-2}, \boldsymbol{b}_{k-1}, \mathcal{P}\left[A^{\top} \boldsymbol{\psi}_{1}\right], \boldsymbol{b}_{k+1}, \ldots, \boldsymbol{b}_{n}\right) \\
&=\operatorname{det}(\left.\boldsymbol{b}_{1}, \ldots, \boldsymbol{b}_{k-2}, \mathcal{P}\left[A^{\top} \boldsymbol{\psi}_{1}\right], \mathcal{P}\left[A^{\top} \boldsymbol{\psi}_{k+1}\right], \boldsymbol{b}_{k+1}, \ldots, \boldsymbol{b}_{n}\right) \\
&+\operatorname{det}\left(\boldsymbol{b}_{1}, \ldots, \boldsymbol{b}_{k-2}, \mathcal{P}\left[A^{\top} \boldsymbol{\psi}_{1}\right], \mathcal{P}\left[A^{\top} \boldsymbol{\psi}_{k}\right], \boldsymbol{b}_{k+1}, \ldots, \boldsymbol{b}_{n}\right) \\
&+\operatorname{det}\left(\boldsymbol{b}_{1}, \ldots, \boldsymbol{b}_{k-2}, \mathcal{P}\left[A^{\top} \boldsymbol{\psi}_{k}\right], \mathcal{P}\left[A^{\top} \boldsymbol{\psi}_{1}\right], \boldsymbol{b}_{k+1}, \ldots, \boldsymbol{b}_{n}\right) \\
& \quad+\operatorname{det}\left(\boldsymbol{b}_{1}, \ldots, \boldsymbol{b}_{k-2}, \mathcal{P}\left[A^{\top} \boldsymbol{\psi}_{k-1}\right], \mathcal{P}\left[A^{\top} \boldsymbol{\psi}_{1}\right], \boldsymbol{b}_{k+1}, \ldots, \boldsymbol{b}_{n}\right) \\
&=\operatorname{det}(\left.\mathcal{P}\left[A^{\top} \boldsymbol{\psi}_{2}\right], \ldots, \mathcal{P}\left[A^{\top} \boldsymbol{\psi}_{k-1}\right], \mathcal{P}\left[A^{\top} \boldsymbol{\psi}_{1}\right], \mathcal{P}\left[A^{\top} \boldsymbol{\psi}_{k+1}\right], \mathcal{P}\left[A^{\top} \boldsymbol{\psi}_{k+2}\right], \ldots, \mathcal{P}\left[A^{\top} \boldsymbol{\psi}_{n+1}\right]\right) \\
&+\operatorname{det}\left(\mathcal{P}\left[A^{\top} \boldsymbol{\psi}_{2}\right], \ldots, \mathcal{P}\left[A^{\top} \boldsymbol{\psi}_{k-1}\right], \mathcal{P}\left[A^{\top} \boldsymbol{\psi}_{k-1}\right], \mathcal{P}\left[A^{\top} \boldsymbol{\psi}_{1}\right], \boldsymbol{b}_{k+1}, \ldots, \boldsymbol{b}_{n}\right) \\
&=\operatorname{det}\left(\mathcal{P}\left[\boldsymbol{\psi}_{1}^{\prime}\right], \ldots, \mathcal{P}\left[\boldsymbol{\psi}_{k-2}^{\prime}\right], \mathcal{P}[\boldsymbol{f}], \mathcal{P}\left[\boldsymbol{\psi}_{k}^{\prime}\right], \mathcal{P}\left[\boldsymbol{\psi}_{k+1}^{\prime}\right], \ldots, \mathcal{P}\left[\boldsymbol{\psi}_{n}^{\prime}\right]\right)=O\left(\prod_{\substack{j=0 \\
j \neq k-1}}^{n}\left\|\hat{A}^{j}\right\|\right) .
\end{aligned}
$$

By similar logic, $\operatorname{det} D_{n}$ is $O\left(\prod_{j=0}^{n-1}\left\|\hat{A}^{j}\right\|\right)$. Thus the $k$ th term in (5.1) - the term multiplied by $A^{\top} \boldsymbol{\psi}_{k}$-is of order $O\left(\left\|\hat{A}^{n}\right\| /\left\|\hat{A}^{k-1}\right\|\right)$. But this term is multiplied by $A^{\top} \boldsymbol{\psi}_{k}=\mathcal{O}\left(\hat{A}^{(k-1) \top} \tilde{\boldsymbol{f}}\right)$, hence

$$
\mathcal{L}[\boldsymbol{v}]-\boldsymbol{f}=\sum_{k=1}^{n} \mathcal{O}\left(\frac{\left\|\hat{A}^{n}\right\|}{\left\|\hat{A}^{k-1}\right\|} \hat{A}^{(k-1) \top \tilde{\boldsymbol{f}}}\right)=O\left(\left\|\hat{A}^{n}\right\|\right) \sum_{k=1}^{n} \mathcal{O}\left(\frac{\hat{A}^{(k-1) \top} \tilde{\boldsymbol{f}}}{\left\|\hat{A}^{k-1}\right\|}\right)=\mathcal{O}\left(\left\|\hat{A}^{n}\right\| \mathbf{1}\right),
$$

and the theorem follows from Corollary 3.2 .

Q.E.D.

Remark: The $k$ th entry in the diagonal matrix $W$ is of the same asymptotic order as the norm of the $k$ th column of $\mathcal{P}\left[A^{\top} \Psi\right]$, and hence also of the $k$ th column of $B$. Thus factoring $B$ into $P+G W$ results in $G$ containing every element of the same order as the norm of its column, and $P$ containing everything of lower order. This decomposition can be achieved by using symbolic algebra in the general case, and by construction in the specific case. 

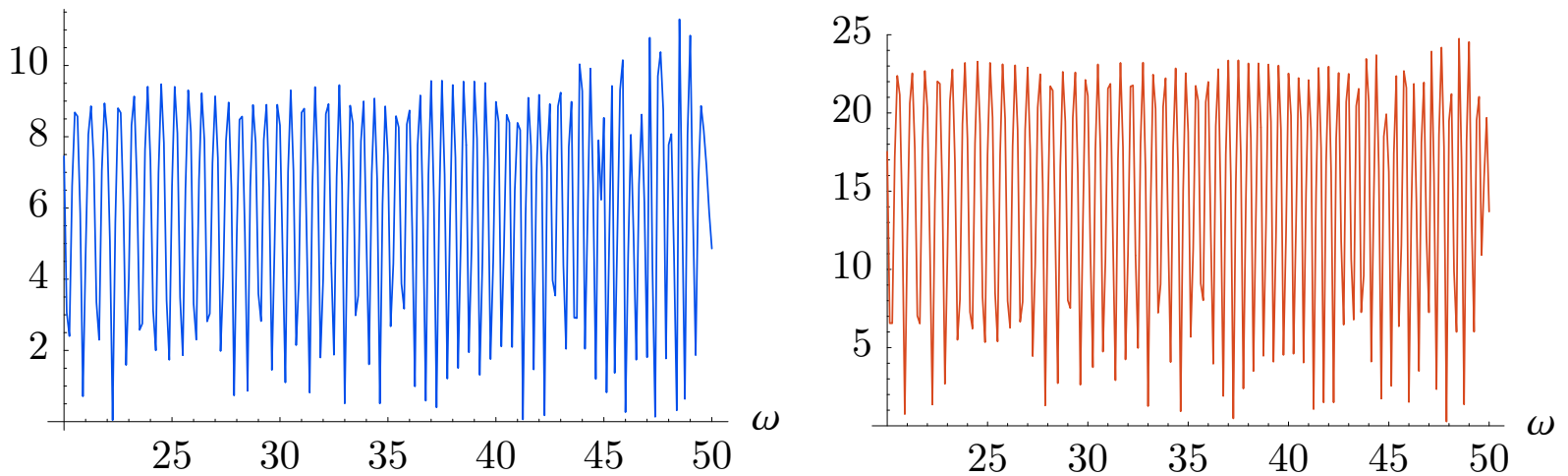

Figure 5: The error scaled by $\omega^{31 / 4}$ of $Q^{B}[\boldsymbol{f}]$ with endpoints for nodes and multiplicities both one (left figure), compared to $Q_{5}^{A}[\boldsymbol{f}]$ (right figure), for $I[\boldsymbol{f}]=\int_{1}^{2}\left[\cos x \mathrm{Ai}(-\omega x)-\omega \mathrm{e}^{x} \mathrm{Ai}^{\prime}(-\omega x)\right] \mathrm{d} x$.
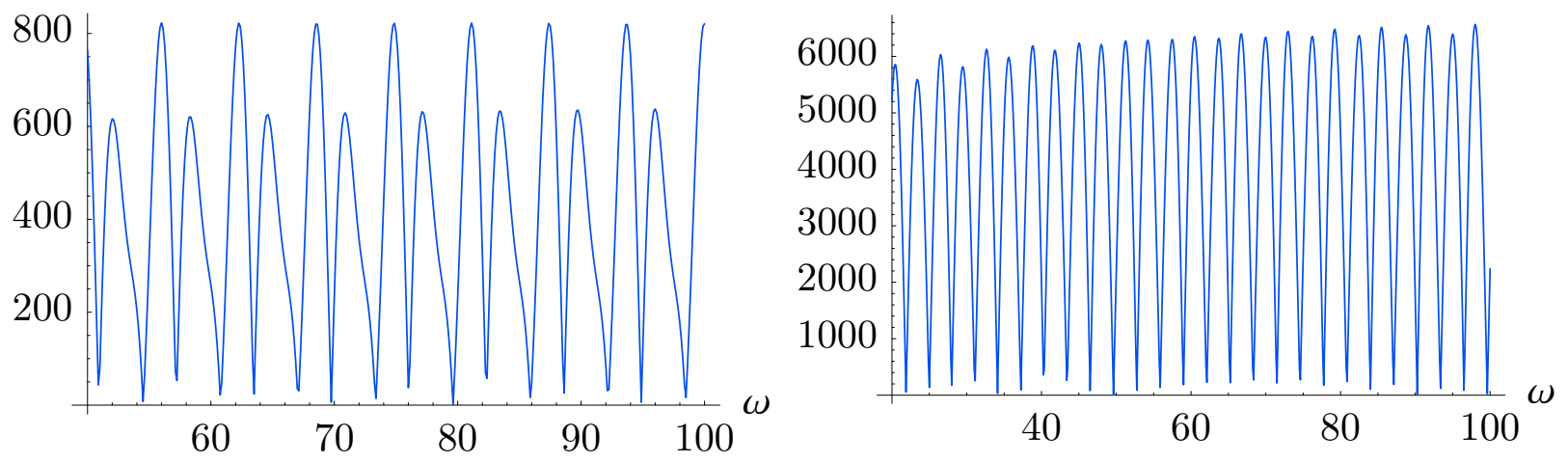

Figure 6: The error scaled by $\omega^{13 / 2}$ of $Q^{B}[\boldsymbol{f}]$ with endpoints for nodes and multiplicities both one (left figure), compared to the error scaled by $\omega^{17 / 2}$ of $Q^{B}[\boldsymbol{f}]$ with an additional node at the midpoint with multiplicity one (right figure), for $\int_{1}^{2}\left[x^{-2} J_{2}(\omega x)+x^{3} J_{3}(\omega x)\right] \mathrm{d} x$.

We once again return to the example from Figure 1. Consider the case with only the endpoints for nodes and multiplicities both one. Then $n=4$, i.e., the dimension times the number of nodes, and the theorem predicts an error of order

$$
O\left(\left\|\hat{A}^{4}\right\| \mathbf{1}^{\top} \hat{A}^{2} \boldsymbol{y}\right)=O\left(\omega^{-6}\left(\omega^{-3}, \omega^{-3}\right)\left(\begin{array}{c}
\omega^{-1 / 4} \\
\omega^{5 / 4}
\end{array}\right)\right)=O\left(\omega^{-31 / 4}\right) .
$$

For comparison, in order to obtain the same order of error we would have needed to take $s$ equal to five in the asymptotic expansion, or a Levin-type method with multiplicities equal to four at the endpoints, resulting in having to solve a much larger system of $4 \cdot 2 \cdot d=16$ equations. Figure 5 confirms the order of error, and compares the error to that of the asymptotic expansion of the same order.

Figure 6 demonstrates that adding a node to $Q^{B}[\boldsymbol{f}]$ does indeed increase the asymptotic order, using the integral from Figure 4. In this case, $\left\|\hat{A}^{n}\right\|=O\left(\omega^{-n}\right)$, hence adding a single node increases the order by two. Note that, because of the large difference in the scaling factor, the errors in the right-hand figure are in fact smaller than those in the left-hand figure.

Remark: The derivatives required to find each $\boldsymbol{\psi}_{k}$ can quickly become unmanageable when either $\boldsymbol{f}$ or $A$ is even moderately complicated. This issue can be mitigated since it is possible to show that including the first $k$ of these basis functions, along with any other basis functions of your choice, results in an error of order $O\left(\left\|\hat{A}^{k}\right\| \mathbf{1}^{\top} \hat{A}^{s+1} \boldsymbol{y}\right)$. In short, adding even just the single, trivially computed, basis function $\boldsymbol{\psi}_{1}=A^{-\top} \boldsymbol{f}$ will increase the asymptotic order. It may also be possible to use finite differences in place of derivatives, in a similar vein to [4], though this idea has not been thoroughly investigated. 


\section{Closing remarks}

Highly oscillatory integrals can be efficiently computed when an appropriate method is used. Using a generalization of an asymptotic expansion, the accuracy of the approximation in fact improves as the frequency of oscillations increases. A Levin-type method retains the asymptotic behaviour of the expansion, while increasing the accuracy of the approximation for fixed frequency. Higher order approximations can be achieved by using multiplicities or the asymptotic basis.

There are still several open questions. The first is whether similar techniques can be used for multivariate highly oscillatory functions. It may be possible to combine the techniques from this paper and [8], which derived a Levin-type method for integrals of the form

$$
\int_{\Omega} f(\boldsymbol{x}) \mathrm{e}^{\mathrm{i} \omega g(\boldsymbol{x})} \mathrm{d} \boldsymbol{x},
$$

to compute integrals whose highly oscillatory component satisfies a partial differential equation. Another open question is handling integrals which contain a turning point, for example

$$
\int_{0}^{1} f(x) \operatorname{Ai}(-\omega x) \mathrm{d} x .
$$

The problem results from $A$ becoming singular, hence the derivation of the asymptotic expansion is no longer valid. The case of $\mathrm{e}^{\mathrm{i} \omega g}$ was handled in $[\mathbf{2}]$ by going to the complex plane, and integrating along a path that approximated the path of steepest descent. However, the asymptotic order depended on using Gauss-Laguerre quadrature, which exploits the exponential nature of an oscillator, which will not work for the Airy oscillator case.

\section{References}

[1] Abramowitz, M., and Stegun, I., Handbook of Mathematical Functions, National Bureau of Standards Appl. Math. Series, \#55, U.S. Govt. Printing Office, Washington, D.C., 1970.

[2] Huybrechs, D., and Vandewalle, S., On the evaluation of highly oscillatory integrals by analytic continuation, SIAM J. Num. Anal., to appear.

[3] Iserles, A., and Nørsett, S.P., Efficient quadrature of highly oscillatory integrals using derivatives, Proceedings Royal Soc. A. 461 (2005), 1383-1399.

[4] Iserles, A., and Nørsett, S.P., On quadrature methods for highly oscillatory integrals and their implementation, BIT 44 (2004), 755-772.

[5] Levin, D., Analysis of a collocation method for integrating rapidly oscillatory functions, J. Comput. Appl. Maths 78 (1997), 131-138.

[6] Olver, F.W.J., Asymptotics and Special Functions, Academic Press, New York, 1974.

[7] Olver, S., Moment-free numerical integration of highly oscillatory functions, IMA J. Num. Anal. 26 (2006), 213-227.

[8] Olver, S., On the quadrature of multivariate highly oscillatory integrals over non-polytope domains, Numer. Math., to appear.

[9] Stein, E., Harmonic Analysis: Real-Variable Methods, Orthogonality, and Oscillatory Integrals, Princeton University Press, Princeton, NJ, 1993.

[10] Xiang, S., Numerical analysis on fast integration of highly oscillatory functions, BIT, to appear. 